Review

\title{
An Introduction to Spiral Steroids
}

\section{Fred Chasalow ${ }^{1,2}$}

\author{
1 Chief Scientist, IOMA LLC; Belmont, CA \\ 2 Visiting Professor, Dept. Lab. Med., VAMC, San Francisco, CA \\ * Correspondence: fchasalow@gmail.com; Tel.: 650-576-1800
}

\begin{abstract}
In addition to the classical steroids, which have cholesterol as a precursor, there are steroids with 7-dehydrocholesterol as a precursor. This review describes the identification of these steroids, their biosynthesis and some aspects of their function. There are three classes of these compounds, distinguished by the number of their carbon atoms, 23, 24 and 25. Each class has a spiral steroid and is a phosphodiester. Up until these investigations, no spiral steroids or steroid phosphodiesters were known. There are at least 13 compounds of which six have been purified to near homogeneity. Each one has been characterized by its mass and proposed composition. They function by regulating the NaK-ATPase. Based on the tissues in which they have been detected, each class of compound seems to regulate a different isoform of the NaK-ATPase. This is an important site of endocrine regulation.
\end{abstract}

Keywords: Spiral steroids; Ionotropin; digoxin-like materials; NaK-ATPase; potassium sparing diuretics

\section{Introduction}

Intracellular fluids have $\mathrm{K}+$ levels of $100 \mathrm{mM}$. Extracellular fluids, including plasma, have $\mathrm{K}+$ levels of 4-6 mM. Thus, there must be a mechanism to 'pump' $\mathrm{K}+$ into the cells against that gradient. In the 1950's, Szent-Gyorgyi proposed that there was an endogenous equivalent to digoxin [1]. His speculation unleashed a 60-year search for the material which, he proposed, might function similar to digoxin. There have been several false candidates, primarily digoxin, itself, ouabain, and more recently, marinobufagenin $[2,3$, 4]. However, in vertebrates, no precursors or metabolites were identified for any of the candidates. Within the last few years, we have identified 13 steroid phosphocholine diesters. These can be divided into three classes, based on the number of carbon atoms in the steroid fragment, and all can be derived from a common precursor. Some, but not all of them, bind to digoxin specific antibodies. Altogether, the discovery points to a new path of investigation to understand $\mathrm{K}+$ regulation. This has consequences for many diseases, especially diseases of pregnancy.

\subsection{What is a spiral steroid?}

A carbon atom can have 4 substituents. If each pair is part of a ring, it would be a spiral steroid. The spiral carbon atom would be a bridge between the rings, but no bond would be part of both rings. What distinguishes a spiral steroid from other bridge carbons is that the planes formed by the two rings are perpendicular. All four of the substituents need not be carbon atoms. To date, carbon atom 17 is the only natural, spiral carbon atom known to be present in steroid molecules. Some synthetic potassium sparing diuretics, including spironolactone and eplerenone, are also spiral steroids. Figure 1 illustrates these features. 


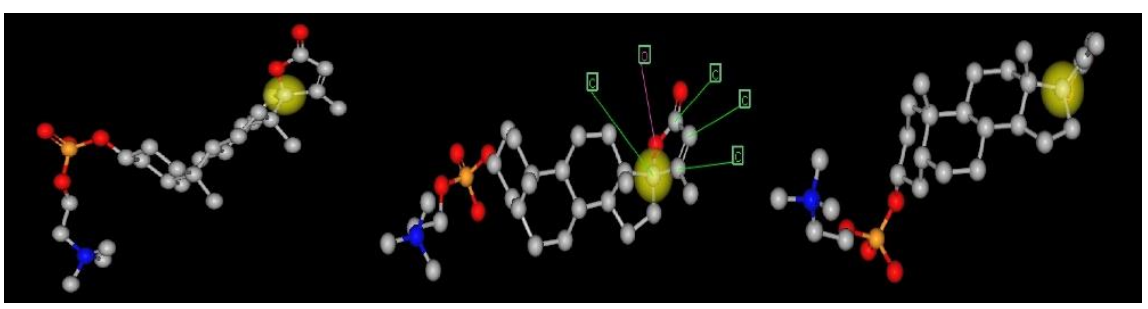

(a)

(b)

Figure 1. Three representations of a spiral steroid - C341.

This figure shows three representations of C341. The yellow ball is on carbon 17, the spiral carbon atom. The view in Panel A is face on to the E-ring and edge on to the B-C-D gonane rings; Panel $\mathrm{C}$ is face on to the $\mathrm{B}-\mathrm{C}-\mathrm{D}$ gonane rings and edge on to the E-ring; Panel B is intermediate in orientation. Panel B also has arrows pointing to the five atoms that form the E-ring.

\subsection{Naming, Symbols and Abbreviations:}

Other than the apparent precursor, 17a-hydroxy-pregna-5,7-dienolone, none of these compounds had been described [5, 6]. Thus, there are no widely recognized, common names. Chasalow selected IONOTROPIN for the name of the first spiral steroid isolated because it seemed to be needed for regulation of ion transport [7]. In retrospect, kaleotropin might have been a better selection. All of the papers published from our laboratory have included Ionotropin as a keyword. This practice has made it possible for interested investigators to find all of the original papers.

There are no common names for steroids with 23, 24, or 25 carbon atoms. Rather than using cross reaction with an antibody specific to a particular cardiotonic glycoside, we established the practice of using the molecular mass of the steroid fragment, as observed in the positive ion mass spectrum, as the basis for the symbol [8]. Note that the apparent mass of a fragment might not be the same as the true formula mass.

Finally, all of the compounds are phosphodiesters. For phosphocholine compounds we preface the observed mass with C; for phosphethanolamine compounds, the preface is $\mathrm{E}$; if the phosphodiester was not identified, the letter is an $\mathrm{X}$ [9].

\subsection{Mass spectra of steroid phosphocholine diesters:}

There must be two breaks in the gonane portion of a steroid in order to generate fragments. As a consequence, steroids usually generate simple mass spectra. In contrast to the classical steroid hormones, the phosphodiesters usually have more than one molecular ion present, depending on the counter cation associated with the phosphate [7]. For the phosphocholine diester, the biggest ion peak is the fragment derived from phosphocholine at $\mathrm{m} / \mathrm{z}=184 \mathrm{Da}$.

Each phosphocholine steroid diester can generate 5 different $\mathrm{m} / \mathrm{z}$ cations, which were designated S, A, B, C, \& D [10]. Figure 2 has an example. Table 1 shows the positive ions that derive from each of the phosphocholine diesters. The relative amounts of $S, A$, $B, C$, and D are dependent on [i] the source of the sample, [ii] the electrolytes present and [iii] how the sample has been extracted or purified. This pattern has also been useful in identifying steroid phosphodiesters in crude extracts [10]. A further complication occurs becauses the phosphoethanolamine compounds don't fragment the same way as the phosphocholine compounds.

\subsection{Steroid Ethanolamine Phosphodiesters (SEP)}

SEP were detected in adrenal and ovarian extracts but not in serum [7]. On the basis of this localization, SEPs seem to be precursors, rather than metabolites, of the phosphocholine steroids. Apparently, the SEP are storage forms and are converted to the phosphocholine diesters by $\mathrm{N}$-methylation. $\mathrm{N}$-methylase is an ACTH-dependent 
enzyme and also converts norepinephrine to epinephrine. Thus, in response to ACTH, [a] norepinephrine could be converted to epinephrine - leading to increased glycolysis and $[b]$ SEP could be converted to a phosphocholine steroid - leading to increased potassium in the heart - increased calcium - and increased blood flow. The combination of events would be important in both the stress response and also during parturition [9].

\subsection{Mass spectral analysis of Steroid Ethanolamine Phosphodiesters (SEP)}

SEP have a different fragment pattern on mass spectrum analysis than that observed with phosphocholine steroids. Because there is no trimethylamine, SEP can't fragment with loss of trimethylamine fragment - the Type A cations. Instead, the $S$ fragment ions for SEP are formed in higher relative intensity. Whereas the phosphocholine fragment at $\mathrm{m} / \mathrm{z}=184 \mathrm{Da}$ is a major ion in spectra from phosphocholine steroids, SEP do not generate the corresponding fragment at $m / z=142 \mathrm{Da}$.

\section{Biochemistry of spiral steroids}

In the 1950's, cardiotonic glycosides were proposed as a substitute for an endogenous potassium sparing hormone [1] and a synthetic potassium sparing diuretic, spironolactone, was discovered [11]. However, no one was successful in preparing a tissue extract that had the same function as spironolactone. In the 1970's, to assist physicians in monitoring possible toxicity of digoxin, an RIA for digoxin was developed [12]. From time to time, prior to initiation of therapy, patients were identified that had unknown materials that were detected by the assay [13]. The material was given a trivial name, digoxin-like-material, which was abbreviated as DLM.

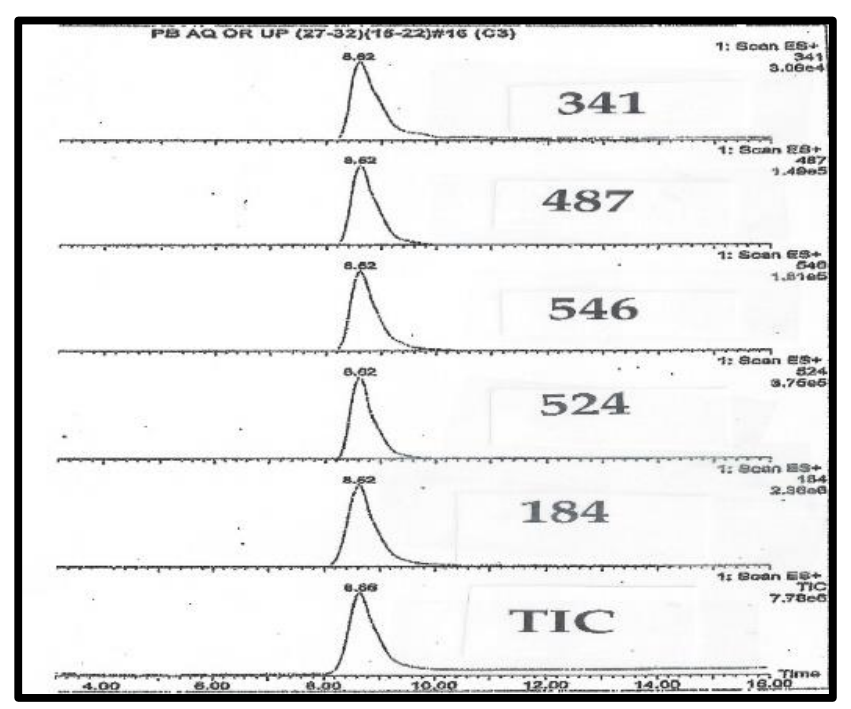

Figure 2. LC-MS chromatography of purified C341 isolated from pig blood.

HPLC chromatograms monitored by the mass ions characteristic of C341. The takeaway lesson from Figures 2 and 3 is all of the expected ions characteristic of C341 coeluted and there were no significant unexplained ions. In total, the two figures suggest a high degree of purity for C341.

Figures 2 \& 3 from Chasalow, F.; Pierce-Cohen, L. Steroids 2018, 136: 63-75 [7]. 


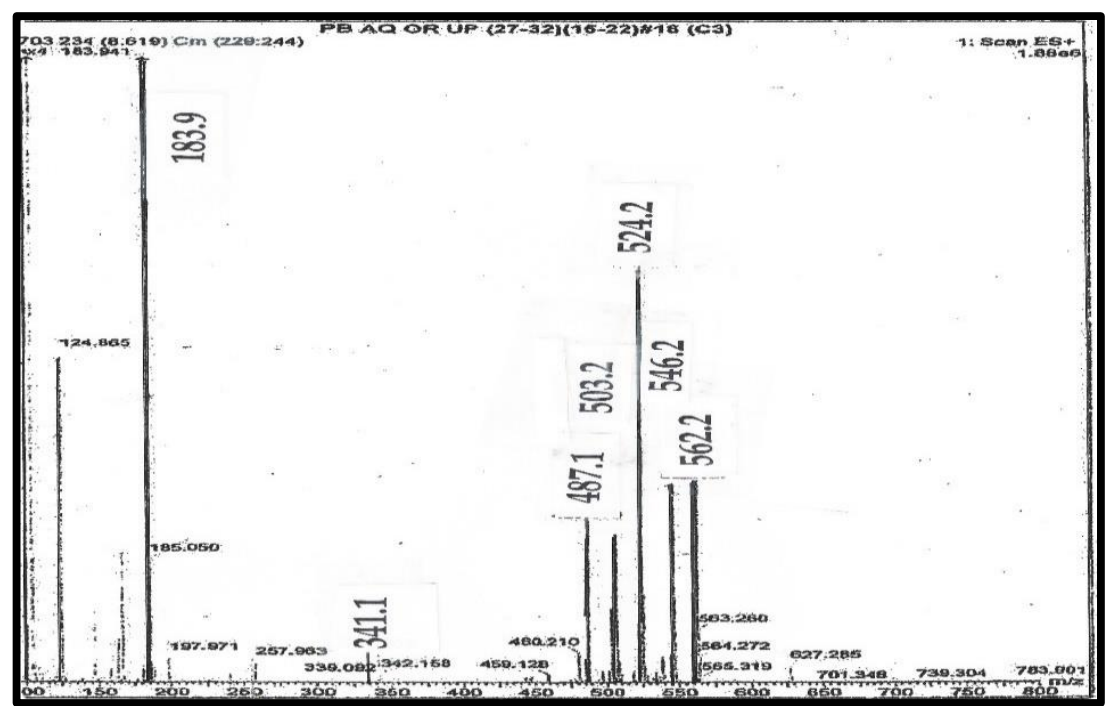

Chasalow,F.; Pierce-Cohen,L. Steroids, 2018, 138: 63-75. [7]

Figure 3. Mass Spectrum of purified C341 from Pig Blood

This is the mass spectrum of C341 as shown on line 5 of Table 1. [i] S is the ion at $341 \mathrm{Da}$; [ii] $\mathrm{A}$ is the ion at $487 \mathrm{Da}$ - generated by loss of trimethylamine from C; [iii] B is the ion at $524 \mathrm{Da}$ as the $\mathrm{H}+$ ion; [iv] C is the ion at 546 as the Na+ion Da; [v] D is the ion at 562 $\mathrm{Da}$ as the K+ ion; The Ion at $503 \mathrm{Da}$ is the loss of trimethylamine from D.

\subsection{Initial candidates for the DLM}

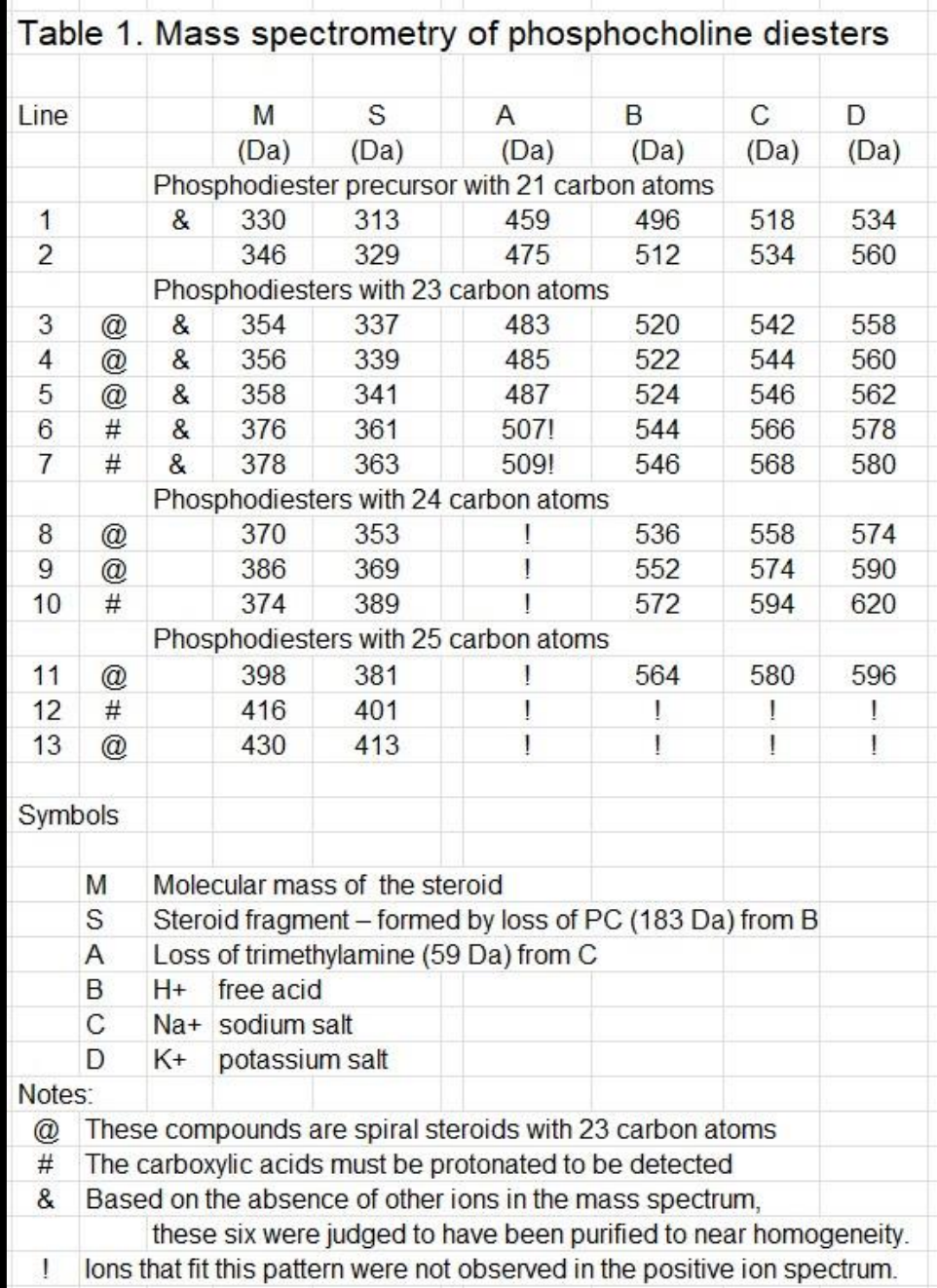


Although the levels of DLM are undetectable (less than $0.05 \mathrm{ng} / \mathrm{ml}$ ) in sera from normal individuals, there are more than 200 papers describing DLM levels in various diseases [14]. Several teams attempted to isolate the DLM. First 'to market' were claims for digoxin [2]. Second, Hamlyn isolated $13 \mu \mathrm{g}$ of 'ouabain' from 80 liters of plasma [3]. There is now a long list of papers describing Endogenous Ouabain $[15,16]$ and a shorter list describing EO as fantasy [17]. A third candidate is marinobufagenin [18]. There is an RIA for marinbufagenin that detects an unidentified substance in human serum from pregnant women [19]. Note that these three cardiotonic steroids are poisons and, in serum samples, have only been detected by RIA. No precursors are known and there are no logical biosynthetic pathways proposed in mammals.

\subsection{How did we find the spiral steroids:}

Our discovery path began with two investigations in the 1980s. First, Chasalow laboratory observed that patients with Smith-Lemli-Opitz Syndrome (SLO) [a] had a steroid disorder, $[\mathrm{b}]$ were potassium wasting and [c] benefited from digoxin therapy [20]. Second, Bradlow laboratory observed that some human breast cyst fluids had high potassium levels [21]. Together, we speculated that the cyst fluids had a steroid hormone that the infants needed, but didn't make. Chasalow measured 'digoxin' in both types of samples [22]. Whatever the material detected with the RIA for digoxin was, a DLM was present both in breast cyst fluids and in serum from healthy newborn infants, but it was not present in the serum from newborn infants with SLO syndrome [20].

\subsection{Why weren't the spiral steroids found prior to these investigations:}

The DLM levels in many breast cyst fluids were over $0.6 \mathrm{ng} / \mathrm{ml}$ [22]. As part of normal patient care, the fluids were aspirated. This was the initial source of the efforts to isolate DLM. First, we confirmed that the DLM was not digoxin (or ouabain) by difference in solubility. Second, we learned to isolate and concentrate the DLM. This could not be done before because methods to isolate either digoxin or classical steroids cannot be used to concentrate DLM. After several false starts, suitable isolation methods were developed. As the properties of DLM are different from that of all other steroids, new methods were needed for purification. [7]

\subsection{How did we isolate the phosphocholine steroid diesters}

Breast cyst fluids with high potassium levels were the first source we used to isolate the DLM. Separation was achieved by HPLC with a gradient of increasing polarity. Repeated chromatography led to two compounds -(a) compound with a UV absorption at $240 \mathrm{~nm}$ that was not a DLM and (b) a DLM with little UV absorbance over $210 \mathrm{~nm}$. However, as the average volume of fluid in a human breast cyst is less than 2 $\mathrm{ml}$, it was difficult to use as a source for isolation of an unknown steroid-like material.

In the second stage, we obtained outdated blood plasma from the local blood collection center and, with the methods developed for the cyst fluid extract, we were able to distinguish 4 steroid phosphodiesters. In the third stage, we obtained 10 liters of porcine blood. In total, with that starting material, we obtained about $10 \mathrm{mg}$ each of 4 pure phosphocholine steroids diesters - C313, C337, C339, and C341. MS ion peaks for the four compounds are listed in Table 1 [7].

\subsection{Determining the chemical formula of the steroid}

Chasalow applied a trial-and-error (T\&E) method to determine the molecular formula of the steroid. There is no a priori reason why only one formula would satisfy the basic rules of chemistry. Once there was a logical composition and with the knowledge of steroid biochemistry, it was possible to propose a structure. The Appendix shows the T\&E analysis of each phosphodiester, its proposed composition and the structure most consistent with steroid enzyme biochemistry. Table 2 summarizes the various features. The proposed structures do not eliminate isomers and stereoisomers. If Occam's Razor does not apply, then there would have to be more unknown 
intermediates, enzymes and pathways. For example, C313 and C329 differ by 16 Da, as would be expected for an extra hydroxy group. This was confirmed by the fragmentation showing loss of 18 Da for C329. It doesn't identify the site of the hydroxy group, but carbon atoms 18, 19, and 21 are eliminated because a hydroxy group on those carbons wouldn't fragment by loss of a molecule of water (18 Da), as was actually observed for C329 and C369.

\subsection{Biosynthesis of steroid ethanolamine phosphate diester - Part 1- the phosphate} Both choline and ethanolamine are required nutrients in mammals [23]. The first step is the condensation of $17 \alpha$-hydroxy-pregna-5,7-dienolone with a phosphate donor.

\begin{tabular}{|c|c|c|c|c|c|c|c|c|c|c|c|}
\hline Table 2 & \multicolumn{11}{|c|}{ Structural components of newly discovered steroids } \\
\hline \multirow[t]{2}{*}{ Symbol } & & \multicolumn{3}{|c|}{ Alkenes } & \multirow[t]{2}{*}{$-\mathrm{OH}$} & \multirow[t]{2}{*}{$-\mathrm{COOH}$} & \multirow[t]{2}{*}{ Spiral } & \multicolumn{4}{|c|}{ Composition } \\
\hline & & 5 & 7 & 23 & & & & C & $\mathrm{H}$ & 0 & Delta \\
\hline \multicolumn{12}{|c|}{21 Carbon atom compounds } \\
\hline C313, E313 & & $x$ & $x$ & & & & & 21 & 30 & 3 & 7 \\
\hline C329, E329 & & $\mathrm{x}$ & $x$ & & $x$ & & & 21 & 30 & 4 & 7 \\
\hline \multicolumn{12}{|c|}{23 Carbon atom compounds } \\
\hline C337, E337 & & $x$ & $x$ & $x$ & & & $\mathrm{x}$ & 23 & 30 & 3 & 9 \\
\hline C339, E339 & & $\mathrm{x}$ & & $\mathrm{x}$ & & & $\mathrm{x}$ & 23 & 32 & 3 & 8 \\
\hline C341, E341 & & & & $x$ & & & $x$ & 23 & 34 & 3 & 7 \\
\hline C361, E361 & & $x$ & & & & $x$ & & 23 & 36 & 4 & 6 \\
\hline C363, E363 & & & & & & $x$ & & 23 & 38 & 4 & 5 \\
\hline \multicolumn{12}{|c|}{24 Carbon atom compounds } \\
\hline X353 & & $x$ & & $x$ & & & $x$ & 24 & 34 & 3 & 8 \\
\hline X369 & & $x$ & & $\mathrm{X}$ & $x$ & & $x$ & 24 & 34 & 4 & 8 \\
\hline C389, E389 & & $x$ & & $x$ & $x$ & $\mathrm{x}$ & & 24 & 36 & 5 & 7 \\
\hline \multicolumn{12}{|c|}{25 Carbon atom compounds } \\
\hline X381 & & $x$ & & $x$ & & & $x$ & 25 & 34 & 4 & 9 \\
\hline X401 & & $x$ & & $x$ & & $\mathrm{x}$ & & 25 & 36 & 5 & 8 \\
\hline X413 & & $x$ & & $x$ & $x x$ & & $x$ & 25 & 34 & 6 & 9 \\
\hline
\end{tabular}

Basically, there are two alternatives: [a] a transfer from either a phosphocholine lipid or a phosphoethanolamine lipid and [b] de novo, in situ synthesis, perhaps from CDP-serine or phosphatidyl-serine. The serine-steroid phosphodiester would be an anion and would not be detected in the cation mass spectrum. Decarboxylation would form E313 [9] and N-methylation would convert E313 to form C313 [24]. As we detected many Exxx compounds in adrenal extracts, methylation might not occur until after the addition of the extra carbons. This would fit with the hypothesis of steroid phosphoethanolamine diesters as the precursor-storage form for the phosphocholine diesters (Figure 3) [7].

Figure 4. Formation of steroid phosphoethanolamine diesters.

2.4 Biosynthesis of phosphosteroid diesters - Part 2 - the steroid

In 1940, Thomas Wolfe wrote: "You can never go home again." That same principle applies in steroid biochemistry. You can't form a $\Delta 7-8$ sterol once it has been reduced by

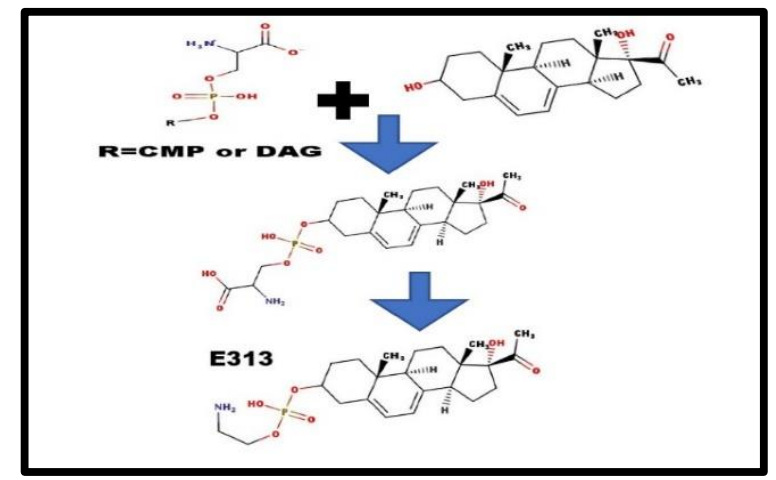

the $\Delta 7$-sterol reductase. UV spectra of both of the steroid phosphocholine diesters with 21 carbon atoms (C313 and C329) are consistent with $\Delta 5-\Delta 7$-dienes. Thus, the patients 
with SLO syndrome (7-dehydrosterol reductase deficiency) provide the 'tell' that the precursor for the phosphodiesters must be a $\Delta 7$-sterol.

After almost a century of steroid investigation, no unconjugated steroids with more than 21 carbon atoms have been described in vertebrates. One could visualize two basic paths to synthesize steroids with more than 21 carbon atoms. One could form them with a [a] different side chain cleavage enzyme from cholesterol and [b] one could add carbons to a precursor with 21-carbon atoms. Burstein, with side chain labeled cholesterol, found only a 6-carbon fragment (25). This confirmed these steroids had to be derived from a 21-carbon precursor. When only phosphocholine steroids with 23 carbon atoms had been identified, the extra carbons could have been added by malonyl coenzyme A (and subsequent decarboxylation).

Fatty acid synthase lenghtens carboxylic acids two carbons at a time. Acetyl coenzyme A is the donor. After condensation, it is dehydrated and the resulting alkene is reduced. Scheme 1 shows how 23 carbon atom phosphosteroids could be synthesized by a fatty acid synthetase-like enzyme. However, that doesn't account for the 24 and 25 carbon atom phosphosteroids.

The three most common acyl coenzyme A derivatives and the corresponding phosphocholine steroids are listed in Table 3. It is possible that other acyl coenzyme A derivatives could also be coupled to C313 or C329. This would depend on the substrate specificity of the fatty acid synthase-like enzyme complex.

Table 3 Origin of the extra carbon atoms in spiral steroids

\begin{tabular}{cclc}
$\begin{array}{c}\text { \# of carbon atoms } \\
\text { in steroid }\end{array}$ & $\begin{array}{c}\text { Acyl- } \\
\text { Co-A }\end{array}$ & $\begin{array}{l}\text { Chemical } \\
\text { Formula }\end{array}$ & $\begin{array}{c}\text { Identifying } \\
\text { Position in steroid }\end{array}$ \\
\hline 23 & Acetyl- & $-\mathrm{CO}-\mathrm{CH}_{3}$ & 22,23 \\
24 & Propyl- & $-\mathrm{CO}-\mathrm{CH}_{2}-\mathrm{CH}_{3}$ & $22,23,24$ \\
25 & Acetoacetyl- & $-\mathrm{CO}-\mathrm{CH}_{2}-\mathrm{CO}-\mathrm{CH}_{3}$ & $22,23,24,25$
\end{tabular}

Note: using this numbering scheme simplifies an understaning of the biosynthesis of all of the spiral steroids. 


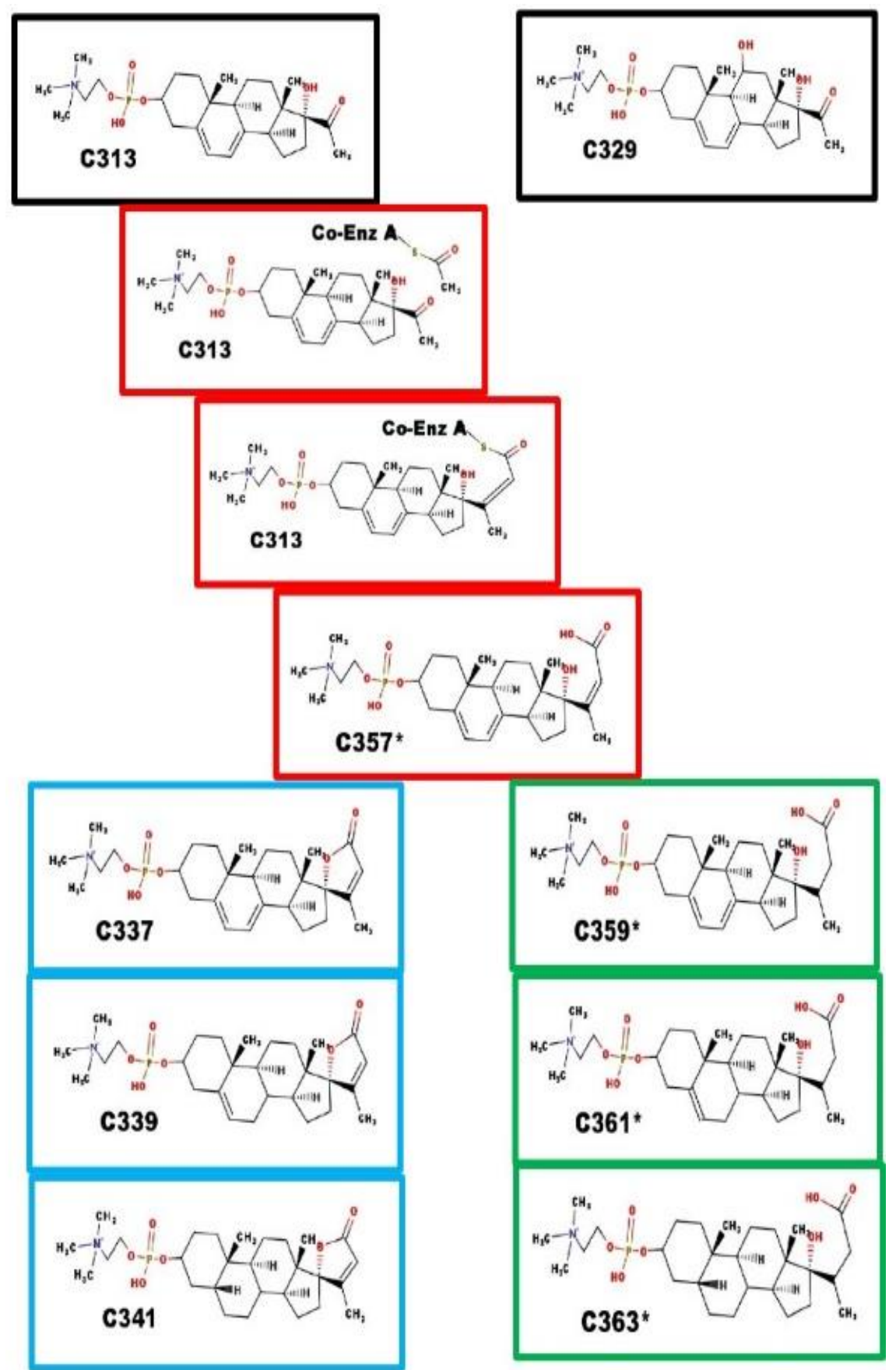

Scheme 1: Enzymes and Biosynthesis of Steroids with 23 carbon atoms

Black boxes - precursor steroids with 21 Carbon atoms; Red boxes - proposed intermediates; Blue boxes - spiral steroids; Green boxes - carboxylic acids. C $357^{*}$ is the branch point. The intermediate steps are similar to the steps leading to lengthening of fatty acids. Ring closure leads to Ionotropin (C341) and DLM. Reduction of the C20-C23 alkene prevents ring closure. The function of $\mathrm{C} 361^{*}$ and $\mathrm{C} 363^{*}$ are unknown at this time.

The extra carbons are added by the action of an unknown enzyme catalyzing a process similar to that of fatty acid synthase - leading to $\mathrm{C} 357^{*}$. C $357^{*}$ is a substrate for two different enzymes: [i] Ring E closure enzyme - leading to the spiral steroids and [ii] C20-C23 reductase leading to the carboxylic acid compounds. The dialkenes of both compounds can be sequentially reduced-first at the $\Delta 7-8$ alkene and then at the $\Delta 5-6$ alkene. The final product is a $5 \beta$-steroid. The $\Delta 5-6$ intermediate also may have a function. 


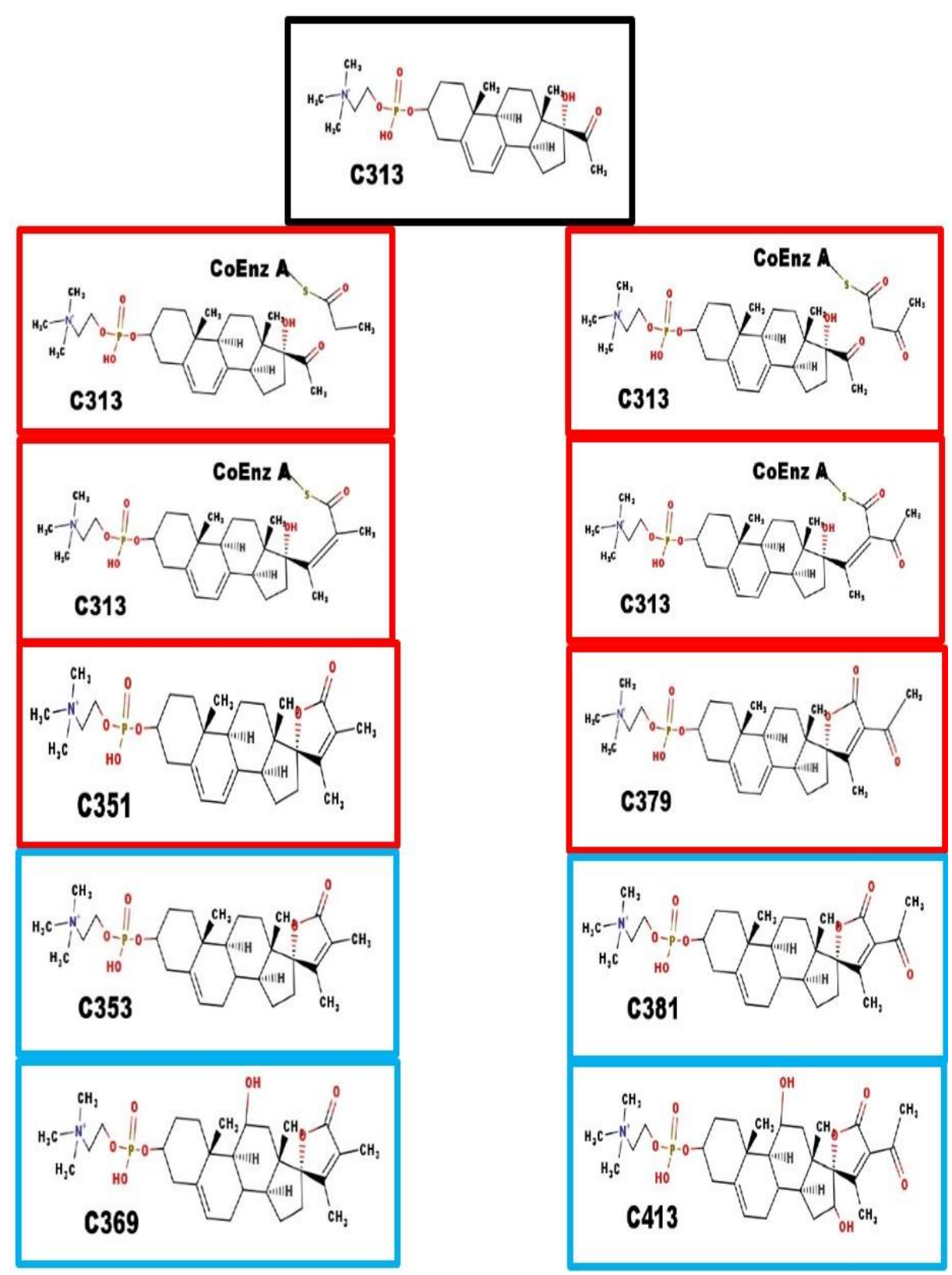

Scheme 2: Enzymes and Biosynthesis of Steroids with 24 and 25 carbon atoms

The fatty acid synthase enzyme complex also seems to accept propyl Coenzyme A (which leads to a steroid with 24 carbon atoms) and acetoacetyl-Coenzyme A(which leads to a steroid with 25 carbon atoms) as a co-substrate with X313.

Black boxes - precursor steroid with 21 carbon atoms; Red boxes - proposed intermediates; Blue boxes - spiral steroids. The intermediate steps are similar to the steps leading to lengthening of fatty acids. C369 fragments with loss of 18 Da which is consistent with the presence of a hydroxyl group. For C369 and C413, we have no evidence at which steps the hydroxyl groups are added nor the carbon atoms to which they are attached. They are shown at carbon 11 and carbon 16 because enzymes with that specificity are known. 


\subsection{Proposed Function of Steroid Phosphocholine Diesters}

Regulation of potassium recovery must occur in every tissue. However, there must be a source of specificity that causes accumulation in only one target tissue. The primary function of the steroid type of potassium sparing diuretics is regulation of the NaKATPase of which there are multiple isoforms. I propose that these match with different classes of steroid phosphodiesters.

Four of the steroid phosphodiesters are likely to have unique functions. Each class has a spiral steroid. Some of the classes also have members with a hydroxy group. The 23carbon atom class also has a carboxyl derivative that doesn't seem to be a precursor and was present in the adrenal extracts. The following sections correlate each class with their proposed endocrine function.

\subsection{Function of the 23-carbon atom class of steroid phosphocholine diesters}

Ionotropin (C341) is the major 23-carbon atom steroid phosphocholine diester. It was isolated on the basis of cross-reaction with digoxin-specific antibodies. The pharmaceutical equivalent, spironolactone, improves renal and heart function in patients with congestive heart failure. 'Endogenous ouabain,' (which was also isolated on the basis of crossreaction with ouabain-specific antibodies) was elevated in patients with essential hypertension [26, 27]. The actual compound measured is probably C341. Whether C341 is the cause of the essential hypertension or part of the attempt to compensate for the hypertension, remains to be determined.

\subsection{Function of the 24-carbon atom class of steroid phosphocholine diesters}

The 24 carbon compounds are C353, C369 and E389*. Steroids of this class were not detected in serum from prepubertal children. E389* was present in bovine adrenal extracts. C369 was present in bovine ovaries. C353 was present in serum from pregnant women. Based on where we found them, this class probably binds to the specific NaKATPase isoforms that are present in gonads and in the fetal-placental unit. Elevated levels of C313 and/or of C329 in serum seem to be a common feature of women with pre-eclampsia [28]. Thus, when fetal potassium is inadequate the placenta secretes both precursors into the maternal circulation, leading to potassium accumulation and maternal hypertension [29]. C369 was present in the serum of obligate heterozygotes for 7-dehydrosterol reductase deficiency [30]. This suggests that C369 (11-hydroxy, 23-methyl-C339) does not cause hypertension. Thus, the theory is the hypertension is caused by the C341 made from the high levels of C313 present in the maternal serum. Elevated C329 could lead to synthesis of C369 and lead to restoration of fetal potassium levels [31].

\subsection{Function of the 25-carbon atom class of steroid phosphocholine diesters}

Scheme 2 also describes the proposed biosynthetic path for the synthesis of steroids with 25 carbon atoms. C381 is the most common member of the 25-carbon atom class. It was found in milk from cattle, goats and sheep. Milk from all three species have high levels of potassium. It was also present in pregnant women, whether or not they had preeclampsia [28]. C413 was detected in fetal calf serum. In addition to binding to NaKATPase, it is possible there is a specific nuclear receptor in mammary glands for a steroid of this class. C381 was also detected in serum from adult males. Its function in males is less obvious.

\subsection{Spiral steroids during pregnancy}

Figure 4 shows representative mass spectra obtained on serum extracts from pregnant women [28]. The study included 40 samples, 20 samples from women with normal blood pressure and 20 samples from women with pre-eclampsia. Miltefosine (sodium hexadecyl phosphocholine) generates an ion at $\mathrm{m} / \mathrm{z}=430 \mathrm{Da}$. As an internal control, $10 \mu \mathrm{l}$ of $0.2 \mathrm{mg} / \mathrm{ml}$ of miltefosine was added to $0.2 \mathrm{ml}$ of serum. For analysis, the ion intensity of each steroid phosphodiester was compared to the intensity of the internal control. If the peak ion intensity of the phosphoester was the same as that of miltefosine, then the 
concentration of the phosphodiester would be about $10 \mathrm{mg} / \mathrm{L}(\sim 20 \mu \mathrm{M})$ of serum. In fact, when C341 was isolated, $10 \mathrm{mg}$ was isolated from 10 liters of porcine blood [7]. Note that in the spectrum from the normotensive woman, the ion intensity of C341 was about 1/5 the intensity of the miltefosine ion.

During pregnancy, all four classes of steroid phosphodiesters are present in serum: (a) 21 carbon class - C313 and C329; (b) 23 carbon class - C341; (c) 24 carbon class - C353; (d) 25 carbon class - X381. There are three different spiral steroids present in maternal serum. Hence, it is not surprising that a single measurement with an immunoassay specific for a cardiotonic glycoside, such ouabain or dixogin, does not adequately reflect fetalmaternal health status.

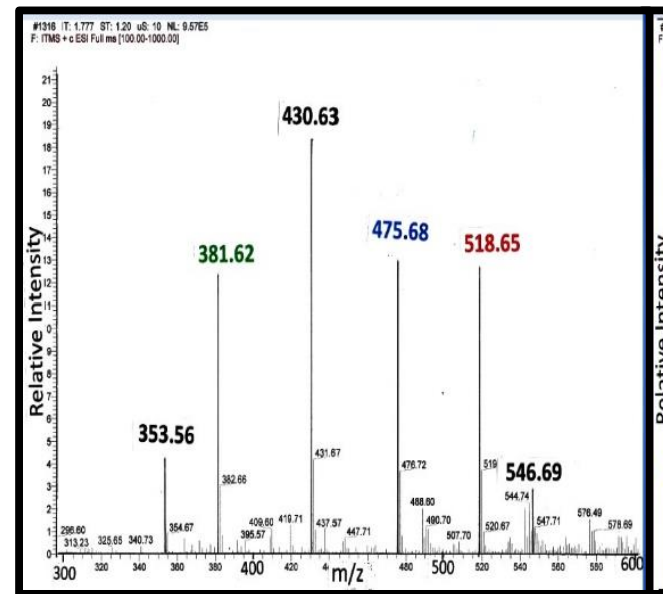

(a)

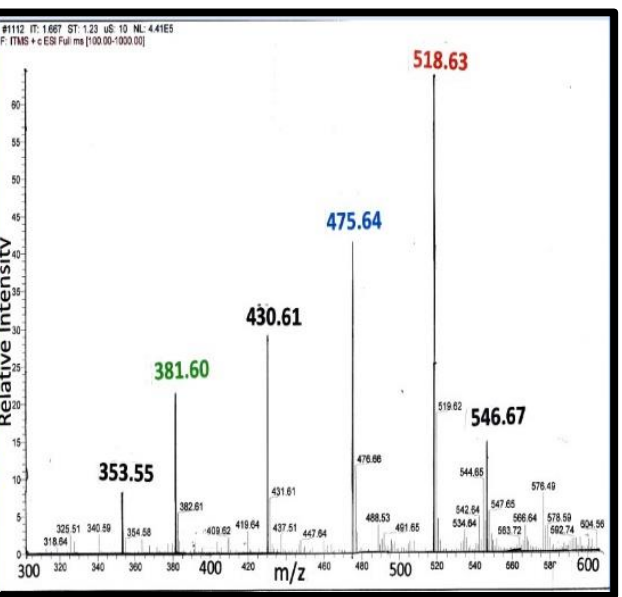

(b)

Chasalow, F.; John, C.; Bochner, R. Steroids. 2019. 151: 108466 [28].

Figure 4: MS from serum steroid phosphodiesters during pregnancy

Panel a: Serum from a normotensive woman at 22 weeks of gestation

Panel b: Serum a woman with pre-eclampsia at 22 weeks of gestation

Peak assignments: (i) 353 Da- C353, (ii) 381 Da- X-381, (iii) 430 Da-Internal control, miltefosine, (iv) $475 \mathrm{Da}-$ Type A ion from C329, (v) 518 Da- type C ion from C313, (vi) 546 DaType C ion from C341.

\subsection{Is there a role for replacement hormone therapy for spiral steroids?}

Patients who benefit from spironolactone and/or eplerenone would be expected to benefit from one of the spiral steroids. The synthetic compounds probably bind to all of the isoforms of NaK-ATPase. Potentially, the natural spiral steroids may be tissue specific and have fewer side effects.

\section{Materials and methods}

This review tries to summarize the discovery of these novel steroids. The details of methods for extraction are in Chasalow [7]. The basic methods for the mass spectroscopy of individual serum samples are in Chasalow, Bochner and John [28].

\section{Conclusions}

Aldosterone was discovered in 1955. Investigators continue to learn more about the role of mineralocorticoides even 70 years after their discovery. The clock has now started on the the biochemical endocrinology of the spiral steroids. The problem is complicated by the fact that, whereas aldosterone functions in a similar fashion in all of its targets, the same is not true for the spiral steroids. C341 (Ionotropin) functions in renal and cardiac tissues, C369 works in gonads and placenta and C381 accumulates potassium in milk in mammary glands. These three compounds are spiral steroid phosphodiesters and bind to different forms of NaK-ATPase. It's as if we considered estrogens, 
androgens and progestogens as all functioning in the same way because they all bind to nuclear receptors even though the receptors are all different.

\section{Appendix}

The appendix contains [i] the Trial and Error table (T\&E) for each of the steroid phosphoesters, [ii] the proposed structures, [iii] specific comments about the proposed structure, and [iv] some information about the tissue in which it was detected.

In the T\&E table, each line illustrates a different possible composition :

- The first column identifies a particular trial.

- Second column selects the number of carbon atoms in the particular trial. The third column has the number of oxygen atoms in the trial.

- To get the $\mathrm{m} / \mathrm{z}$ of the steroid $17 \mathrm{Da}$ must be added to the $\mathrm{m} / \mathrm{z}$ observed of the steroid fragment observed in the mass spectrum. When phosphoesters fragment, the oxygen between the phosphorus and the carbon atom remains attached to the phosphorus atom. Carboxylic acids must be protonated to be detected in the positive ion spectrum. Thus, only 15 Da needs to be added to get the correct $\mathrm{m} / \mathrm{z}$ value if the proposed structure contains a carboxylic acid.

- H-Max is the maximum number of hydrogens that could form a molecule. It is twice the number of carbon atoms plus two. H-Max must be larger than H-Req.

- H-Req in each trial (Hydrogen atoms required to make a molecule) is calculated by subtracting the contributions to the $\mathrm{m} / \mathrm{z}$ from the carbon and oxygen atoms.

- Delta is half the difference between H-Max and H-Req. Delta is the number of double bonds or rings in the proposed structure. Each Delta reduces the number of hydrogens required by two atoms. Alkenes, ketones and carboxylic acids each contribute a Delta. Steroids must have Delta greater than 4 because of the four rings in the gonane. Delta must be between 4 and 12 to be consistent with a steroid molecule.

- The next line in the table increases the number of oxygen atoms and repeats the calculation. After the range of possible Delta is exceeded, the next lines increase the number of carbon atoms and the process is repeated.

- Knowledge of steroid enzyme biochemistry is used to restrict choices. For example, Occam's razor suggests C337, C339, and C341 represent step wise reduction of double bonds or ketones. Similarly, MS-MS fragmentation with loss of 18 Da suggests the presence of a hydroxy group. The hydroxy group could not be at carbons 18, 19, or 21 because the adjacent carbon is a tertiary carbon.

- Finally, the bold print identifies the composition that best fits the steroid fragment.

- The proposed composition does not eliminate stereoisomers.

Beneath each T\&E table is the proposed structure.

21-carbon compounds

23-carbon compounds

24-carbon compounds

25-carbon compounds

‘ Carboxylic acid
C313, C329

C337, C339, C341, C361*, C363*

C353, C369, C389*

X381, X401*, X413 


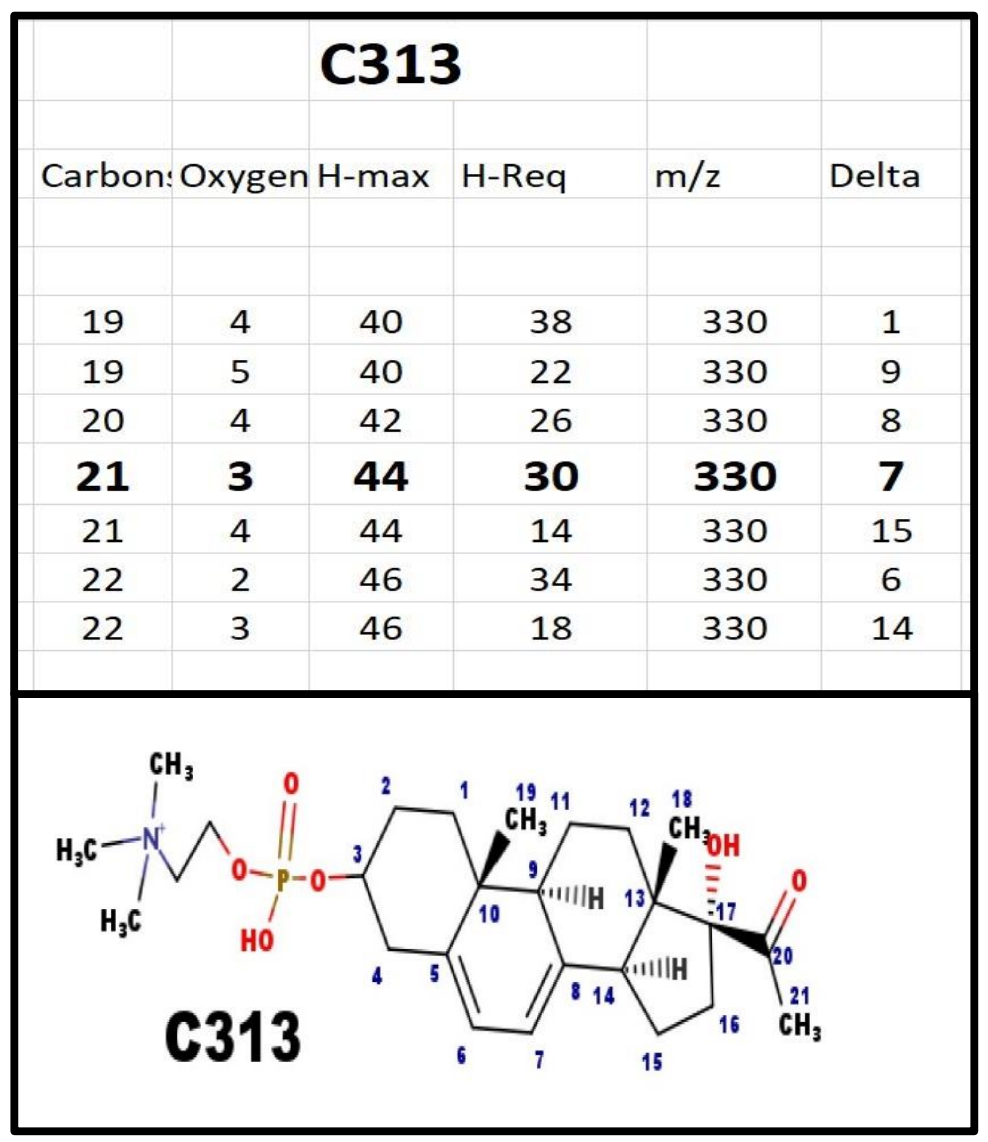

C313 was one of the two phosphosteroids in serum from newborn infants with 7dehydrosterol reductase deficiency (Smith-Lemli-Opitz syndrome). E313 and C313 are the precursors of all of the phosphodiester steroids. 


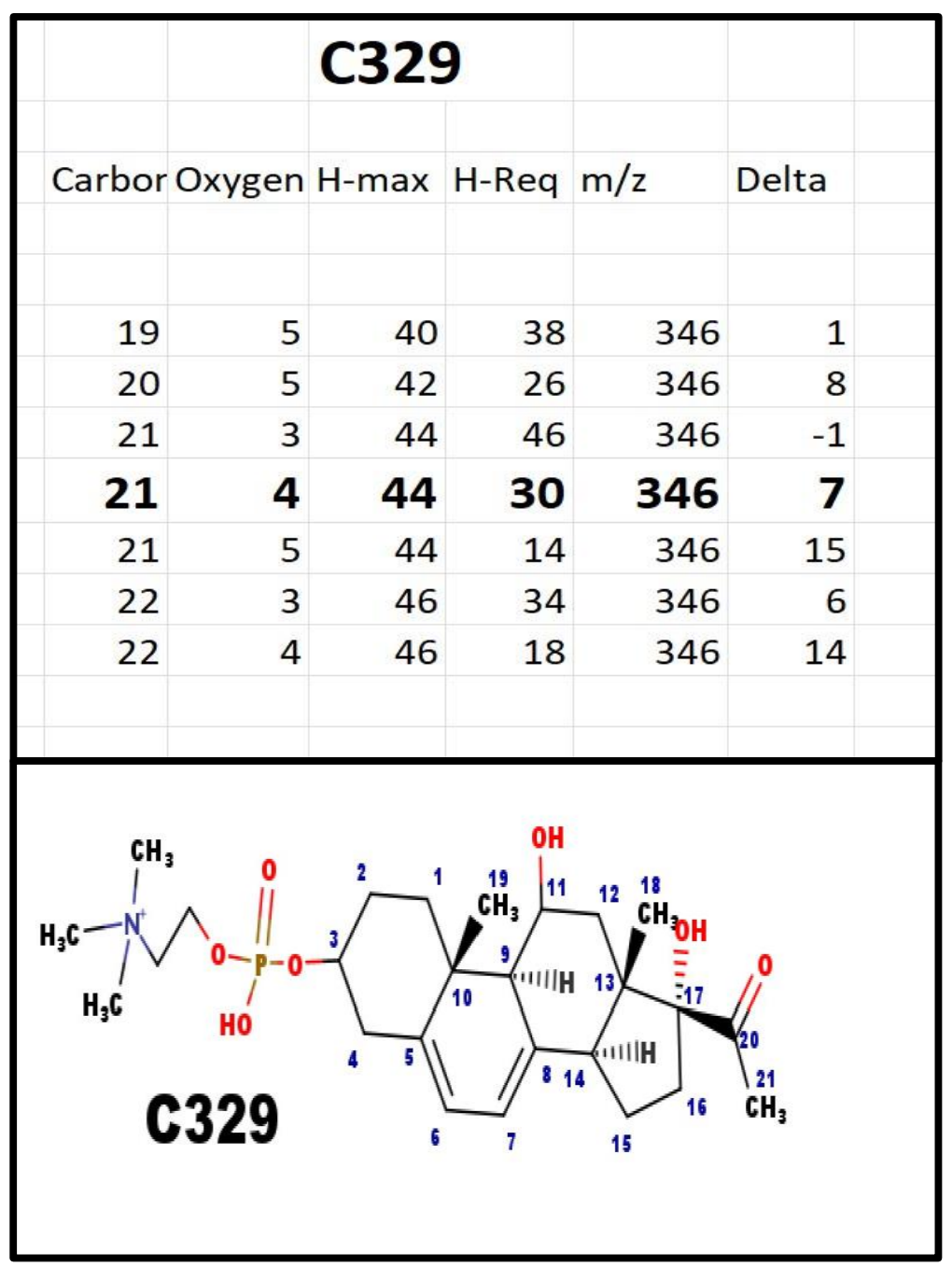

In bovine ovarian extracts, an $\mathrm{m} / \mathrm{z}$ fragment ion was observed at $311 \mathrm{Da}$. There is no logical Delta that could be added to the structure of C313 to get to C311. However, loss of 18 Da would be consistent with $\mathrm{C} 329$. The $\mathrm{Na}+$ ion of the intact molecule would have an $\mathrm{m} / \mathrm{z}=534 \mathrm{Da}$ and the $\mathrm{Na}+$ salt fragment with loss of $59 \mathrm{Da}$ (trimethylamine) to produce an ion at $475 \mathrm{Da}$. Both were observed in serum extracts from pregnant women but not from extracts of plasma from children prior to puberty. 


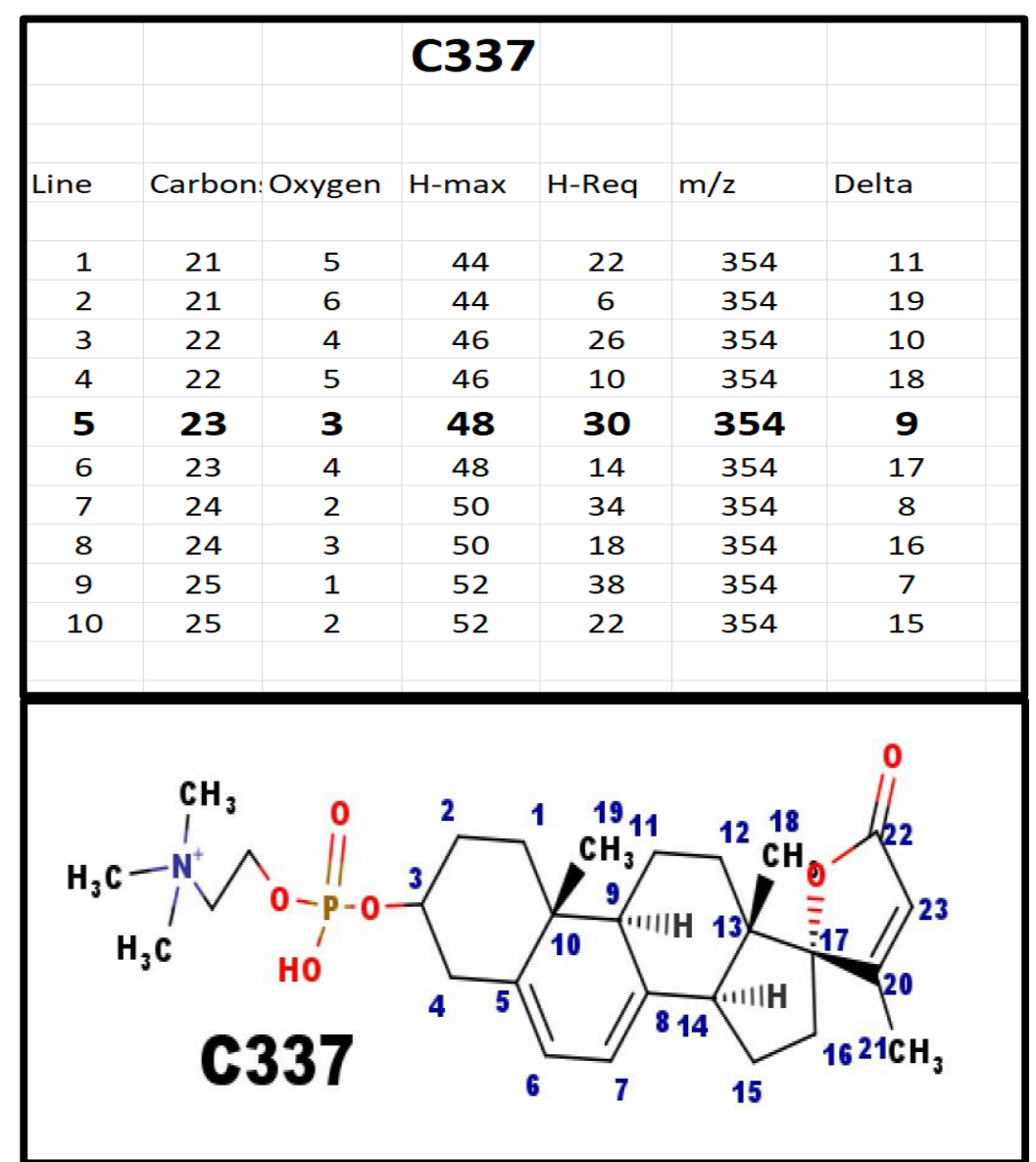
scheme.

This is the first spiral steroid phosphodiester formed in the proposed biosynthetic 


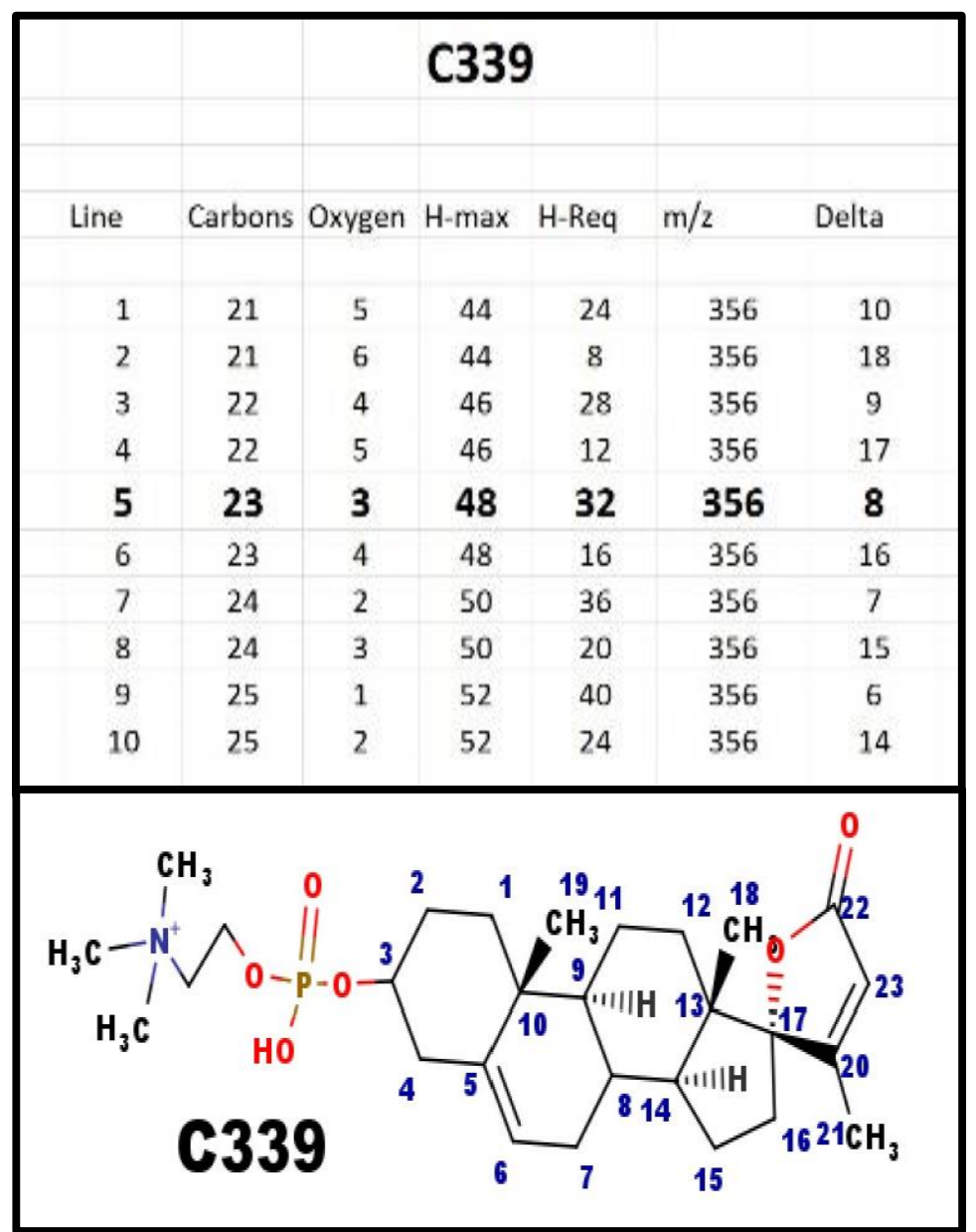

C339 was one of the first group of steroid phosphodiesters that we isolated from mammalian and avian serum. Although C341 is the primary 23-carbon spiral steroid in mammals, the primary 23-carbon spiral steroid in both chickens and turkeys is C339.

After adrenarche, the human weak androgen is DHEA-S, which is a $\Delta 5-6$ androgen just like the proposed structure of C339. Thus, human NaK-ATPase needs to be able to discriminate the spiral steroid from the weak androgen. Both chickens and turkeys have much lower levels of their weak androgen, which is not DHEA=S, and it doesn't compete with the spiral steroid for the usual NaK-ATPase isoform. 


\begin{tabular}{|c|c|c|c|c|c|c|}
\hline \multirow[b]{2}{*}{ Line } & & \multirow[b]{2}{*}{ Oxygen } & \multicolumn{2}{|c|}{ C341 } & \multirow[b]{2}{*}{$\mathrm{m} / \mathrm{z}$} & \multirow[b]{2}{*}{ Delta } \\
\hline & Carbon & & H-max & H-Req & & \\
\hline 1 & 21 & 5 & 44 & 26 & 358 & 9 \\
\hline 2 & 21 & 6 & 44 & 10 & 358 & 17 \\
\hline 3 & 22 & 4 & 46 & 30 & 358 & 8 \\
\hline 4 & 22 & 5 & 46 & 14 & 358 & 16 \\
\hline 5 & 23 & 3 & 48 & 34 & 358 & 7 \\
\hline 6 & 23 & 4 & 48 & 18 & 358 & 15 \\
\hline 7 & 24 & 2 & 50 & 38 & 358 & 6 \\
\hline 8 & 24 & 3 & 50 & 22 & 358 & 14 \\
\hline 9 & 25 & 1 & 52 & 42 & 358 & 5 \\
\hline 10 & 25 & 2 & 52 & 26 & 358 & 13 \\
\hline
\end{tabular}

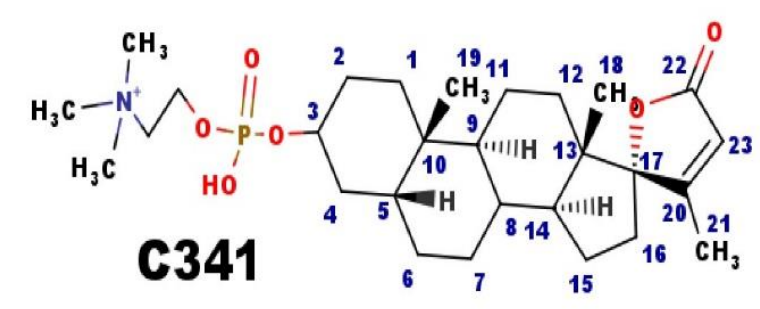

The proposed structure has Delta=7, five for the rings, one for $\Delta 20-23$ alkene and one for the carboxyl group. C341 was isolated on the basis of DLM assays. It wasn't present in serum from infants with Smith-Lemli-Opitz syndrome. The plane of the E-ring is almost perpendicular to the plane of the gonane ring. Note that the AB ring juncture is shown in the $\beta$ configuration. Although testosterone is reduced to the $5 \alpha$-configuration, the substrate for its reduction is the $\Delta 4-3$ ketone. The phosphosteroids would not be substrates for that enzyme. The only enzyme, that reduces the $\Delta 5-6$, forms the $5 \beta$-configuration, similar to the cholic acids. C341, as a $5 \beta$-steroid would not be expected to interact with the androgen receptor. In contrast, spironolactone has a $\Delta 4-3$ ketone and interacts with both the DLM receptor and the androgen receptor.

When C341 was isolated, the starting material was 10 liters of fresh porcine blood. We obtained $10 \mathrm{mg}$ of C341. As an estimate, ignoring all losses during isolation, this would be a serum concentration of $100 \mu \mathrm{g} / \mathrm{dL}$. Normal 17-hydroxyprogesterone levels are $<200$ $\mathrm{ng} / \mathrm{dL}$. Peak cortisol levels are $<25 \mu \mathrm{g} / \mathrm{dL}$ and corticosterone levels are $<3 \mu \mathrm{g} / \mathrm{dL}$. Normal young adult DHEA-S levels at 300-500 $\mu \mathrm{g} / \mathrm{dL}$. Thus, except for DHEA-S, C341, even allowing for losses in isolation, the serum concentration of C341 is the highest of all the steroids. C341 is the primary spiral steroid with 23-carbon atoms in mammals.

We assigned Ionotropin as the trivial name for C341. The name was selected because patients who were deficient had an electrolyte defect, suggesting that it would function to stimulate ion transport. 
The asterisk in the name indicates that the proposed structure is a carboxylic acid

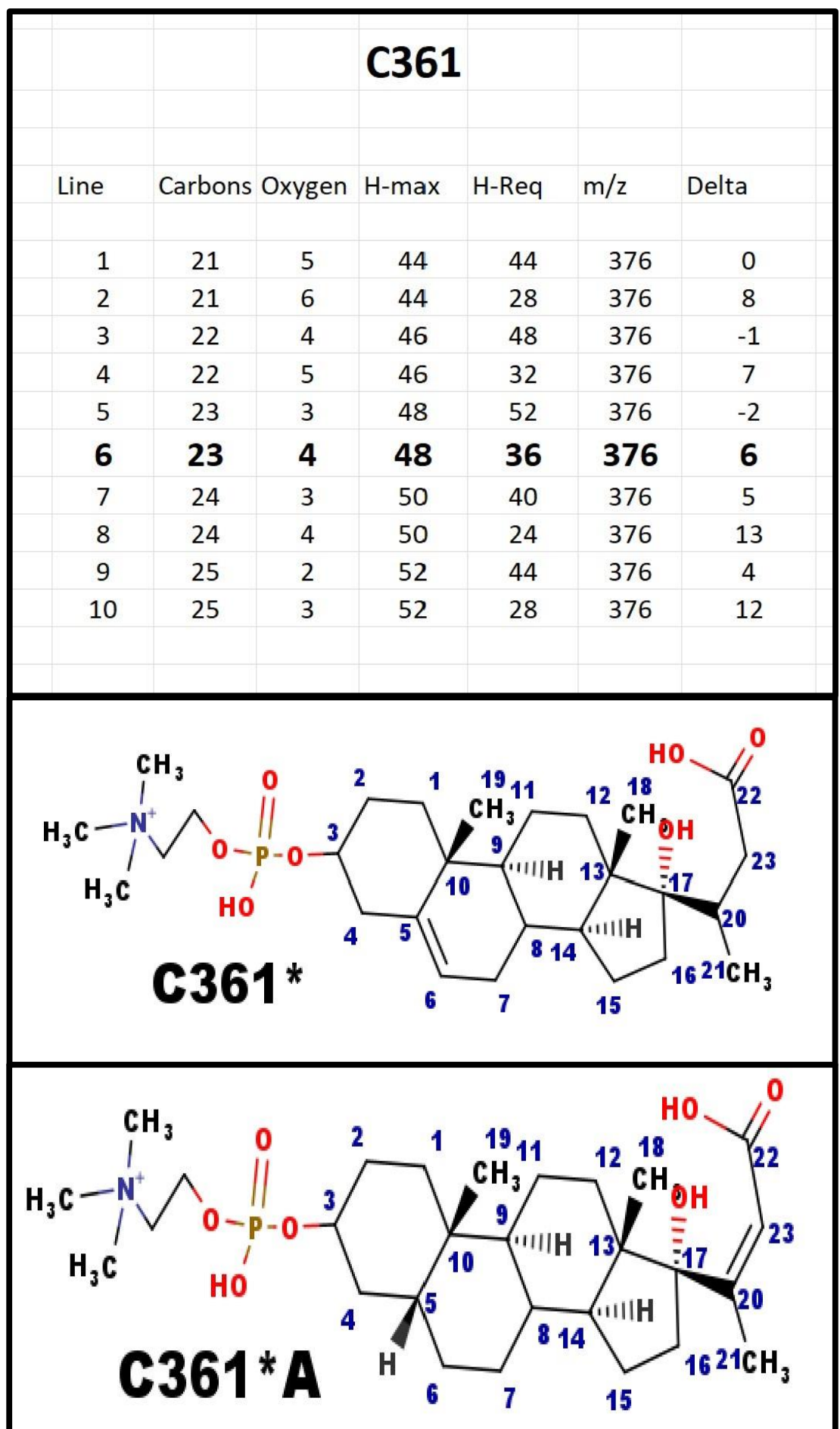

and the apparent mass is actually 2 Da higher than the molecular formula suggests.

There are two isomers that might generate C361 - [1] $\Delta 5-6$ with C20-C23 reduced and [2] $\Delta 20-23$ with C5-C6 reduced. In fact, the mass spectrum that obtained from the sample of C361 that we had isolated seems to show a double peak. Perhaps both isomers were present. In $\mathrm{C} 361^{*} \mathrm{~A}$, the five atoms that form the E-ring are more or less coplanar. Thus, the atoms for E-ring have juxtaposition to form the ring. The same is not true for C361. The reduction of the side chain alkene is similar to the enzyme step in the fatty acid synthase pathway. C361A could be an intermediate in the formation of C341. The same is not true for C361A, which may have another biological function. 


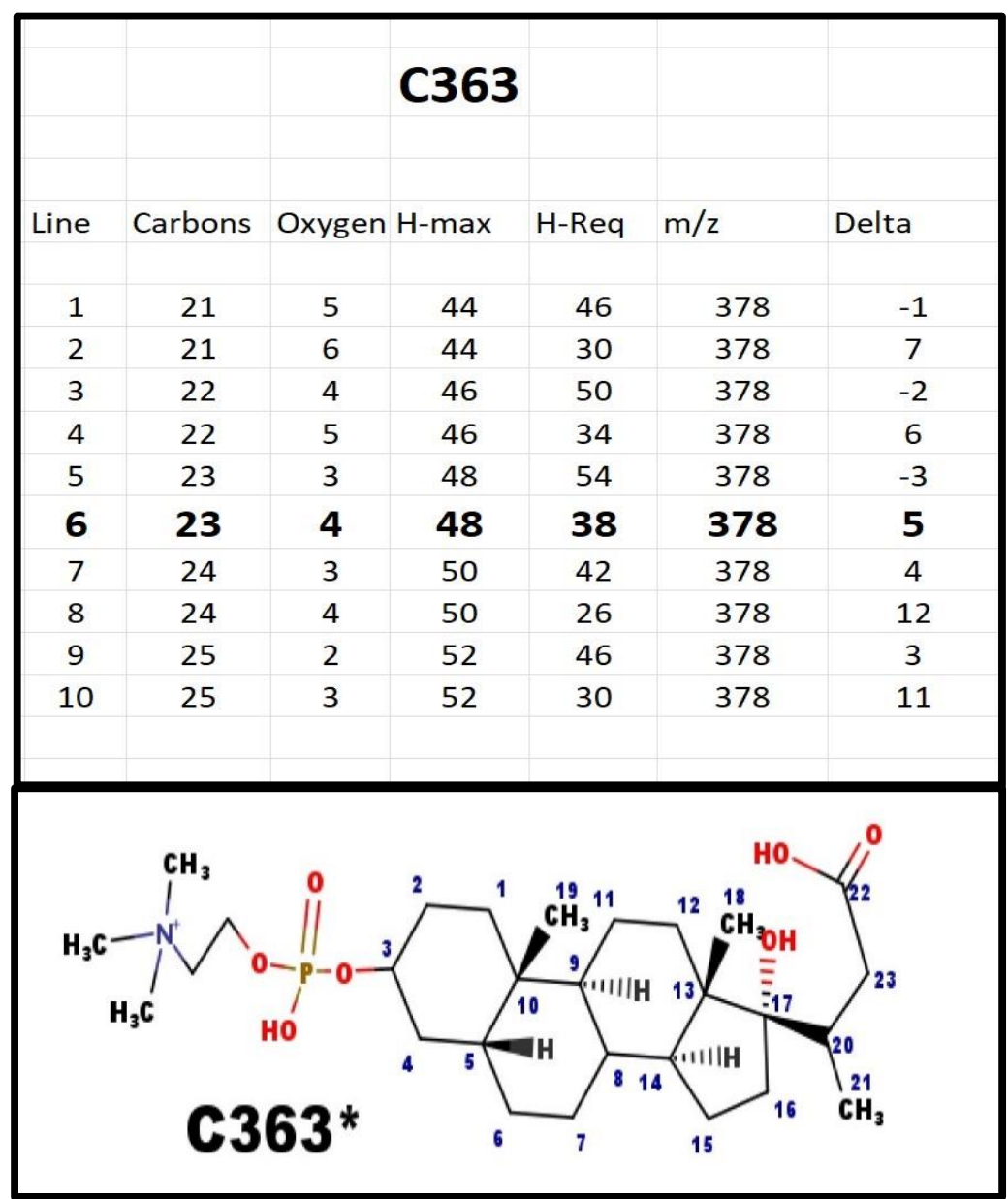

$\mathrm{C} 363^{*}$ must have a Delta of 5 . Four for the rings and one for the carboxyl group. It is the only phosphosteroid we found without a 20-23 alkene. This seems to be an important synthetic branch point. The 3D structure suggests that once the bond is reduced, the lactone E-ring can't form because the 5 atoms are not co-planar. The H-NMR was indistinguishable from the NMR from C341, indicating most of the hydrogen atoms were in the same environment. The localization of this compound in bovine adrenal extracts, rather than in serum, suggests it is not a non-functional metabolite. Small amounts of C363 were also present in extracts from blood obtained from the blood bank.

A comparison of C341 and C363 indicates a difference of $22 \mathrm{Da}$. The $22 \mathrm{Da}$ is composed of three components, [i] $18 \mathrm{Da}$ for the water, [ii] $2 \mathrm{Da}$ to protonate the carboxylate anion and [iii] 2 Da to reduce the C20-C-23 alkene. Thus, C363 is not on the obvious path to synthesize C341. 


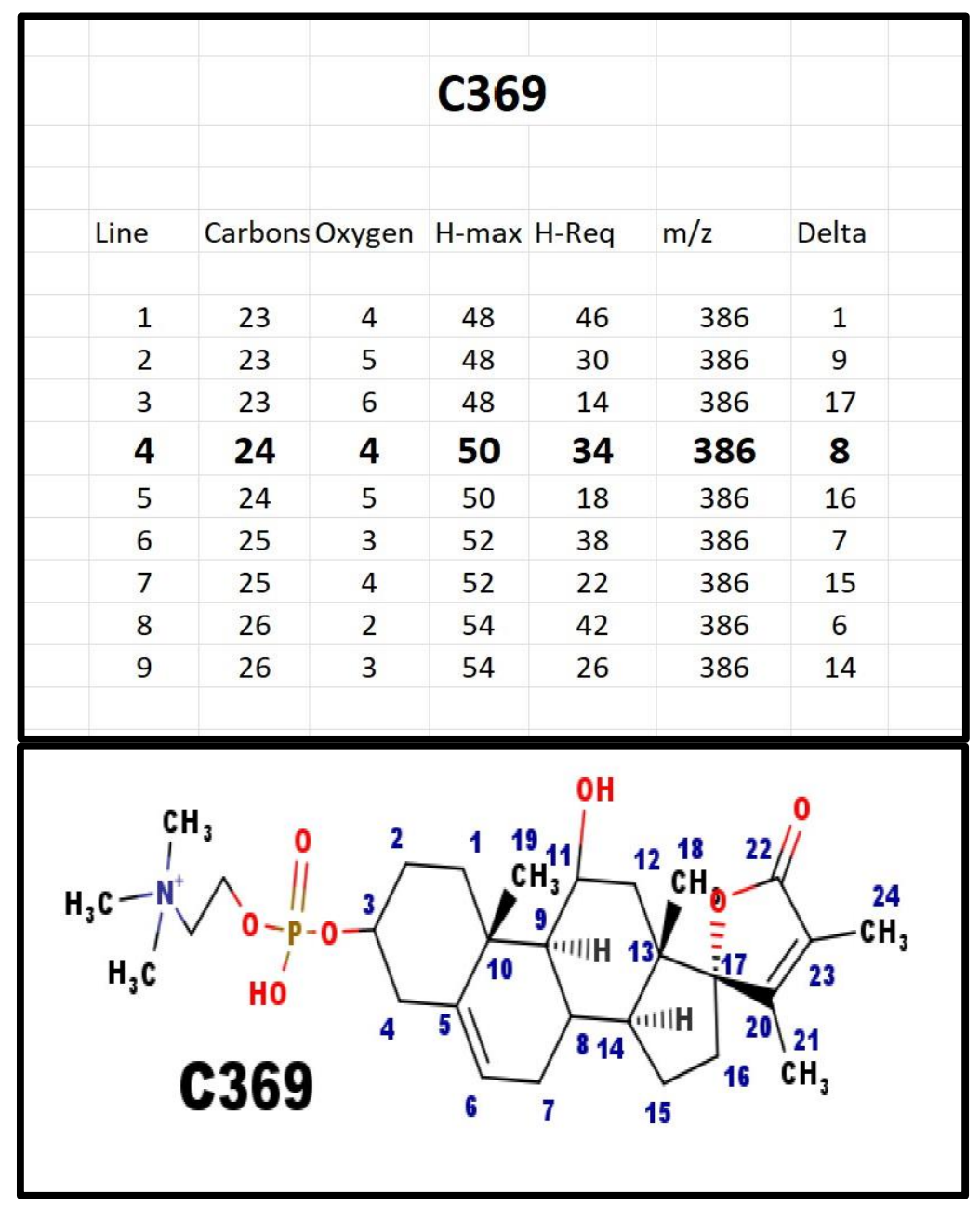

Both C369 (m/z=552 Da) and E369 (m/z=510 Da) were presnt in bovine ovarian extracts. C369 and E369 both fragment with loss of a fragment of $18 \mathrm{Da}$. This would be consistent with a hydroxy group. The precursor for both compounds would be X329, which also fragments with loss of $18 \mathrm{Da}$. If there was a hydroxy on carbon 18, 19, 21, or 24, it would not fragment with loss of water $(18 \mathrm{Da})$ because the adjacent carbon atom can't form an alkene.

10 out of 10 obligate heterozygotes for 7 -dehydrosterol reductase deficiency had high levels of C369 in their serum [30]. In contrast, at 22-24 weeks of gestational age, only 3 of 40 pregnant women had high levels of C369 in their serum [28]. The estimated incidence of the heterozygotes for this disorder is 5-10\%. Perhaps, the high levels of C369 may play a part in the preservation of the gene in the population, despite the consequence to the individuals who are homozygous for a mutation at the gene. 


\section{C381}

\begin{tabular}{|c|c|c|c|c|c|c|}
\hline Line & \multicolumn{2}{|c|}{ Carbons Oxygen } & H-max & H-Req & $\mathrm{m} / \mathrm{z}$ & Delta \\
\hline 1 & 23 & 4 & 48 & 58 & 398 & -5 \\
\hline 2 & 23 & 5 & 48 & 42 & 398 & 3 \\
\hline 3 & 23 & 6 & 48 & 26 & 398 & 11 \\
\hline 4 & 24 & 4 & 50 & 46 & 398 & 2 \\
\hline 5 & 24 & 5 & 50 & 30 & 398 & 10 \\
\hline $\mathbf{6}$ & $\mathbf{2 5}$ & $\mathbf{4}$ & $\mathbf{5 2}$ & $\mathbf{3 4}$ & $\mathbf{3 9 8}$ & $\mathbf{9}$ \\
\hline 7 & 25 & 3 & 52 & 50 & 398 & 1 \\
\hline 8 & 26 & 4 & 54 & 22 & 398 & 16 \\
\hline 9 & 26 & 2 & 54 & 54 & 398 & 0 \\
\hline 10 & 27 & 2 & 56 & 42 & 398 & 7 \\
\hline
\end{tabular}

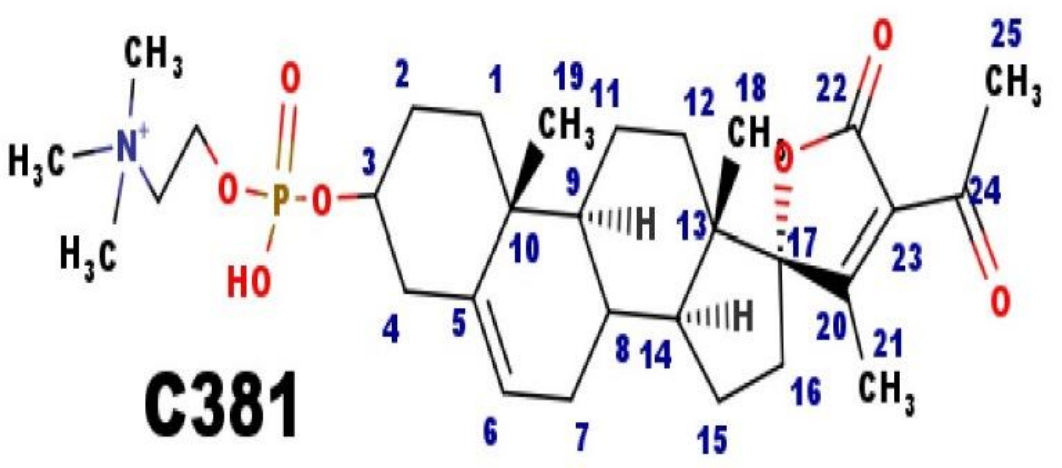

C381 was found in extracts from milk from three species - cows, sheep and goats. No other steroid phosphodiester was present in the extracts. C381 was also present in serum from pregnant women but not in prepubertal children. Note that milk is high in potassium ions. 


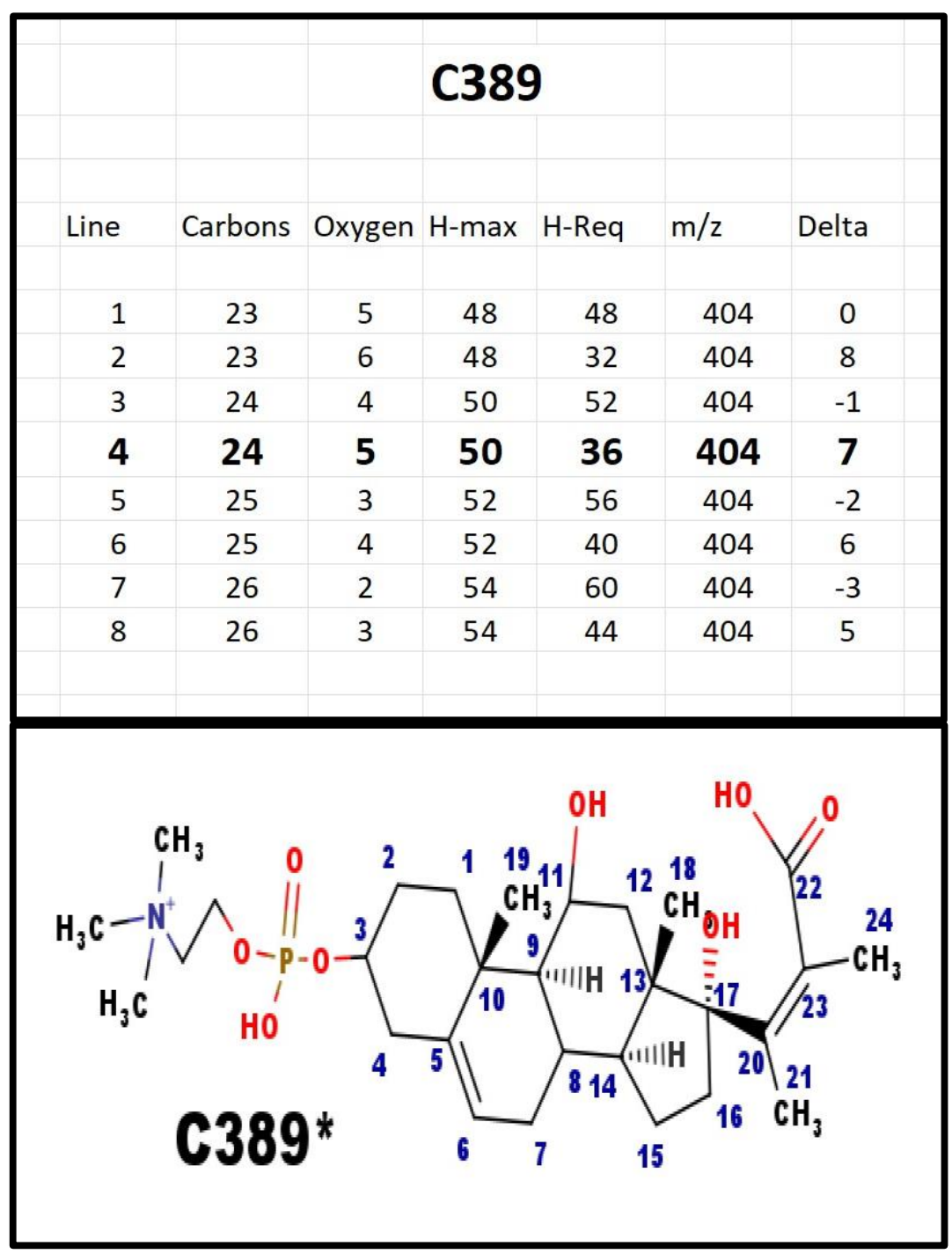

C389 and E389 were both present in extracts from bovine adrenals. Note that C389 and C369 differ by 20 Da even the only difference is loss of molecule of water with 18 Da. We attribute the extra $2 \mathrm{Da}$ as necessary to convert the carboxylate anion to a cation in order for it to be detected in the positive ion spectrum. 


\section{C413}

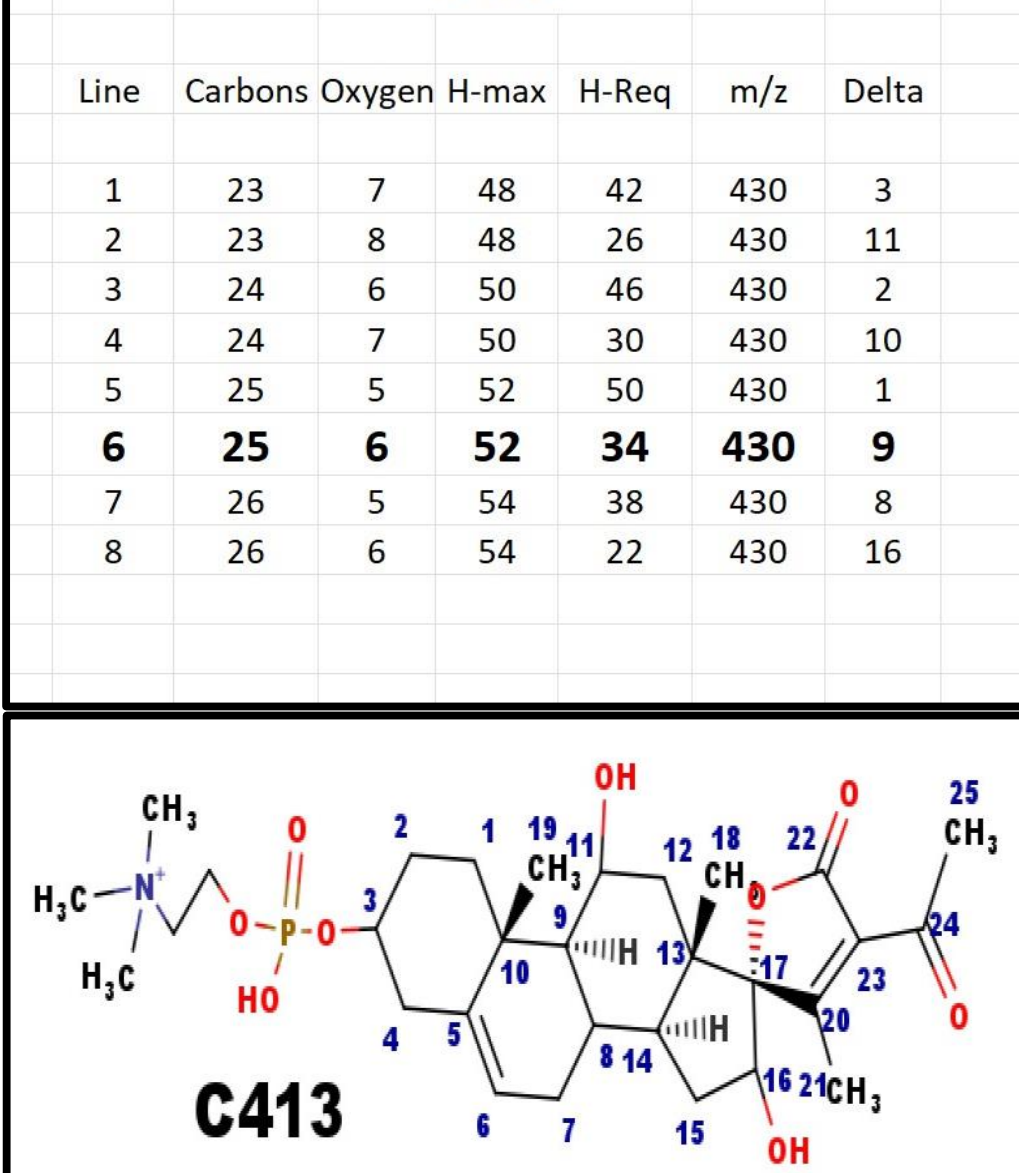

C413 was detected in extracts from fetal calf serum. The T\&E table suggests that there are two extra hydroxy groups compared to C381. However, the site for the hydroxy groups is not defined. The $C$ ion for $C 413$ would have $m / z=596 \mathrm{Da}$. A a cation with that was observed, however, the ion concentration wasn't sufficient for MS-MS confirmation. Funding: This research did not receive any specific grant from funding agencies in the public, commercial or not-for-profit sectors. Dr. Ron Bochner personally funded the pilot study of women with pre-eclampsia. The Smith-Lemli-Opitz Foundation funded the investigation of the patients with SLO syndrome. AMUR Research Corp funded most of the original investigations. Kerix funded the purification of the compounds with 23 carbon atoms and the investigation of the chemical formulas. This work was partially supported by the Research Service of the United States Department of Veterans Affairs. Current support from IOMA LLC.

Institutional Review Board Statement: This paper is a review of publications from many laboratories. The authors of each cited publication complied with Institutional Review Board requirements appropriate at the time of original publication.

Data Availability Statement: There is no new data presented in this article.

\section{Acknowledgments:}

I specifically wish to recognize three very special colleagues: Dr. Ron Bochner, Dr. H. Leon Bradlow and Dr. Sandra Blethen (Chasalow). Colleagues included, Dr. Kathryn King, LIJMC, Dr. Sharon Nachman, LIJMC, Dr. Gary Jarvis, VA Medical Center, San Francisco, CA and Dr. Constance John, VA Medical Center, San Francisco, CA. Dr. John encouraged me and made laboratory space and equipment available. My two laboratory chiefs were Michael Davis and Lori PierceCohen. Dr. Forbes Porter and Dr. Christopher Wassif of the NICHD provided serum samples from patients and obligate heterozygotes with Smith-Lemli-Opitz syndrome. 
Marvin Applets were used for drawing, displaying and characterizing chemical structures and reactions, Product Version 21.1 ChemAxon (https://www. Chemaxon.com).

Conflicts of Interest: The authors declare no conflict of interest.

Sample Availability: No samples of the spiral steroids are available at this time.

\section{References}

1. Szent-Gyorgyi, A. Chemical physiology of contraction in body and heart muscle. Academic Press. New York, NY. (As cited in Labella FS. Endogenous digitalis-like factors: Introductory Remarks. LaBella FS. Fed Proc. 1985, 44: 2780-2781.

2. Shaikh, I.; Lau B,; Siegfried, B,; Valdes, R. Isoalation of Digoxin-like immunoreactive Factors from Mammalian Adrenal Cortex. J Biol Chem 1991, 266: 13672-13678.

3. Hamlyn J.; Blaustein M,; Bova, S.; DuCharme, D.; Harris, D,; Mandel, F.; Mathews, W.; Ludens, J. Identification and characterization of a ouabain-like compound from human plasma. Proc Natl Acad Sci USA. 1991, 88: 6259-6263.

4. Fedorva, O.; Talan, M.; Agalakova, N.; Lakatta, E.; Bagrov, A. Endogenous Ligand of $\alpha 1$ Sodium Pump, Marinobufagenin, is a Novel Mediator of Sodium Chloride-Dependent Hypertension. Circulation 2002, 105:1122-1127. doi: 10.1161/hc0902.104710

5. Slominski, A.; Zmijewski, M.; Semak, I.; Sweatman, T.; Janjetovic, Z.; Li, W.; Zjawiony, J. Sequential metabolism of 7-dehydrocholesterol to steroid 5,7-dienes in adrenal glands and its biological implication in skin. PLOS ONE 2008, 4(2): e4309. doi: 10.1371/journal.pone.0004309.

6. Shackleton, C.; Roitman, E.; Guo, L.; Wilson, W.; Porter, F. Identification of 7(8) and 8(9) unsaturated adrenal steroid metabolites produced by patients with 7-dehydrosterol-delta-7-reductase deficiency (Smith-Lemli-Opitz Syndrome). J Steroid Biochem Mol Biol. 2002, 82: 225-32. . Pubmed/12477489

7. Chasalow, F.; Pierce-Cohen L. Ionotropin is the mammalian digoxin-like material (DLM). It is a phosphocholine ester of a steroid with 23 carbon atoms. Steroids 2018, 136: 63-75. doi:10.1016/j.steroids. 2018.03.001. Epub 2018 Mar.

8. Chasalow, F. Spiral Phosphocholine Steroids and DLM in Chicken Eggs (Gallus gallus domesticus). EC Paediatrics 2019, 8.10. 01-12. doi: 10.31080/ecpe.2019.08.00593

9. Chasalow, F. A New Concept: Ionotropin might be a Factor in Mobilization for [a] the Fight or Flight Response and [b[ Child Birth. Pediatrics (E-cronicon) 2018, 7(9) 909-918. doi: 10.31080/ecpe.2018.07.00341

10. Chasalow, F. Phosphocholine Steroid Esters in Pacific Oysters (Crassostrea gigas). EC Paediatrics 202, 9.11: 115-126. doi: 10.31080/ecpe.2020.09.0084

11. Sabbadin, C.; Calo, L,; Armanini, D. The story of spironolactone from 1957 to now: from sodium balance to inflammation. G Ital Nefrol 2016; 33 (S66) - ISSN 1724-5590 - (C) 2016 Società Italiana di Nefrologi

12. Walsh, P.; Crawford, F.; Hawker, C. Measurement of digoxin by radioimmunoassay. Annals of Clinical and Laboratory Science. 1977, 7: 79-87.

13. Graves S. The Possible role of Digitalislike Factors in Pregnancy-Induced Hypertension. Hypertension. 198710 \{suppl I\}: I-84- I-86.

14. Bagrov, A.; Shapiro, J.; Fedorova, O. Endogenous Cardiotonic Steroids: Physiology, Pharmacology, and Novel Therapeutic Targets. Pharmacological Reviews. 2009, 61: 9-38.

15. Hamlyn, J.; Blaustein, M. Endogenous Ouabain: Recent Advances and Controversies. Hypertension. 2016, 68: 526-532 (2016). doi: 10.1161/HYPERTENSIONAHA.116.06599.

16. Blaustein M. Why isn't endogenous ouabain more widely accepted? ? Am J Physiol Heart Circ Physiol. 2014, 307(5): H635-H639. doi: 10.1152/ajpheart.00404.2014.

17. Nicholls, M.G.; Lewis, K.; Yandle, T.; Lord, G.; McKinnon, W.; Hilton, P. Ouabain, a circulating hormone secreted by the adrenals, is pivotal in cardiovascular disease. Fact or fantasy? J Hypertens. 2009, 27(1),3-8. doi: 10.1097/HJH.0b013e32831101d1.

18. Abi-Ghanem, D.; Lai, X.; Berghman, L.; Horvat, D.; Li, J.; Romo, D.; Uddin, N.; Kamano, Y.; Nogawa, T.; Xu, J.; Petit, G.; Puschett, J. A chemiflourescent immunoassay for the determination of marinobufagenin in body fluids. J Immunoassay and Immunochemistry 2011, 32(1), 331-46 doi: 10.1080/15321819.2010.538107 
19. Lenaerts, C.; Bond, L.; Tuytten, R.; Delporte, C.; Antwerpen, P.; Blankert, P. D3. Early prediction of preeclampsia risk assessment: analytical determination for marinobufagenin in pregnant women. The Journal of Maternal-Fetal \& Neonatal Medicine. 2016, 29: sup2, 18. doi: 10.1080/14767058.2016.1234778

20. Chasalow F, Blethen S, Taysi K. Possible abnormalities of steroid secretion in children with Smith-Lemli-Opitz syndrome and their parents. Steroids. 1985, 46: 827-843.

21. Bradlow H, Fleisher M, Breed C, Chasalow F. Biochemical classification of patients with gross cystic breast disease. N Y Acad Sci. 1990, 586:12-16.

22. Chasalow, F.; Bradlow, HL. Digoxin-like materials in human breast cyst fluids. Ann N Y Acad Sci. 1990, 586:107-16. doi: 10.1111/j.1749-6632.1990.tb17797.x. PubMed PMID: 2162647.

23. Gibellini, F.; Smith, T. The Kennedy Pathway - De Novo Synthesis of phosphatidyl ethanolamine and Phosphatidylcholine. IUBMB Life, 2010, 62 (6): 414-428 (2010). doi: 10 .1002/iub.337

24. Bobenchik, A.; Augagneur, Y.; Hao, B.; Hoch, J.; Mamoun, C. Phosphoethanolamine methyltransferases in phosphocholine biosynthesis: functions and potential for antiparasite therapy. FEMS Microbiol Rev, 2011, 35: 609-619. doi: 10.1111/j.1574-6976.2011.00267.x

25. Burstein, S.; Gut, M. Biosynthesis of pregnenolone. Recent Prog Horm Res. 1971, 27:303-49. doi: 10.1016/b978-0-12-571127-2.50032-8. PMID: 4946132

26. Tentori, S.; Messaggio, E.; Brioni, E.; Casamassima, N.; Simoni, M.; Zagato, L.; Hamlyn, J.; Manunta, P.; Lanzani, C. Endogenous ouabain and aldosterone are co-elevated in the circulation of patients with essential hypertension. J Hypertension 2016, 34, 2074-2080.

27. Manunta, P.; Ferrandi, M.; Bianchi, G.; Hamlyn, J. Endogenous ouabain in cardiovascular function and disease. J Hypertension 2009, 27, 9-18. doi: 10.1097/HJH.obo13e32831cf2c6.

28. Chasalow, F.; John, C.; Bochner, R. Spiral steroids as potential markers for pre-eclampsia: a pilot study. Steroids. 2019. 151: 108466. doi:10.1016/j.steroids.2019.108466. Epub 2019 Jul 26. PubMed PMID: 31351941.

29. Chasalow, F. Pre-eclampsia: It's all about Potassium. In: Eclampsia.

Ed. By Sharon Wright. Nova Science Publishers. Inc. New York. 2021, 63-113. ISBN: 978-1-53619-574-3.

30. Chasalow, F.; Blethen, S. Steroid Metabolic Consequences of 7-Dehydrosterol Reductase Deficiency (SLO). EC Paediatrics. 2020, 9: 60.69. doi: 10.31080/ecpe.2020.09.00720

31. Chasalow,F. Role of Spiral Steroids in Pregnancy and Pre-Eclampsia [Online First], IntechOpen, October 13th 2021. doi: 10.5772/intechopen.100337. Available from:

https://www.intechopen.com/online-first/78858. 